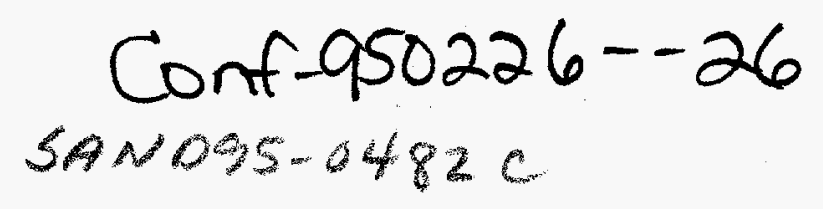

\title{
Polarization characteristics, control, and modulation of vertical-cavity surface emitting lasers
}

\author{
Photonics Research Department \\ Sandia National Laboratories \\ Albuquerque, NM 87185-0603 \\ *AT\&T Bell Laboratories, STC \\ Breinigsville, PA 18031
}

Kent D. Choquette, K. L. Lear, R. P. Schneider, Jr., R. E. Leibenguth, J. J. Figiel, S. P. Kilcoyne, M. Hagerott-Crawford, and J. C. Zolper

\begin{abstract}
The gain-dependent polarization properties of vertical-cavity surface emitting lasers and methods for polarization control and modulation are discussed. The partitioning of power between the two orthogonal eigen polarizations is shown to depend upon the relative spectral alignment of the nondegenerate polarization cavity resonances with the laser gain spectrum. A dominant polarization can thus be maintained by employing a blue-shifted offset of the peak laser gain relative to the cavity resonance wavelength. Alternatively, the polarization can be controlled through use of anisotropic transverse cavity geometries. The orthogonal eigen polarizations are also shown to enable polarization modulation. By exploiting polarization switching transitions in cruciform lasers, polarization modulation of the fundamental mode up to $50 \mathrm{MHz}$ is demonstrated. At lower modulation frequencies, complementary digital polarized output or frequency doubling of the polarized output is obtained. Control and manipulation of vertical-cavity laser polarization may prove valuable for present and future applications.
\end{abstract}

Keywords: vertical-cavity laser polarization, polarization control, polarization modulation.

\section{INTRODUCTION}

Vertical-cavity surface emitting lasers (VCSELs) are rapidly approaching insertion into system applications such as fiber-based optical data links, laser printers, optical displays, or optical scanning systems. Hence it is important to fully elucidate the optical properties of VCSELs, particularly attributes which differ from conventional edge emitting laser diodes. The polarization of VCSEL emission is found to possess several unique differences from its edge emitting counterpart. ${ }^{1-5}$ First, the lasing power in each electromagnetic field distribution can be distributed between two orthogonal linear TE eigen polarizations. ${ }^{2,3}$ Secondly, this pair of eigen polarizations can have a random orientation in the plane of the active region, 1,4 where the orientation can vary between neighboring devices and with temperature. ${ }^{2}$ Finally, higher-order transverse modes tend to emerge orthogonally polarized from the dominant eigen polarization of the fundamental mode. 1,5

Maintaining a constant and consistent polarization state will be critical for VCSELs deployed in polarization-sensitive applications. Several methods to produce a single dominant polarization have been proposed. 6-11 All of the schemes rely in some manner on breaking the symmetry in the plane of the active region, ${ }^{6}$ such as by introducing anisotropic gain 7,8 or loss 9,10 to the laser, or by employing an anisotropic transverse cavity geometry. 11 Fundamentally; what governs the competition between the VCSEL eigen polarizations in the absence of an intentional anisotropy, is unclear. Moreover, complete polarization control of all transverse spatial modes uniformly for all VCSELs in an array has been elusive. 


\section{DISCLAIMER}

This report was prepared as an account of work sponsored by an agency of the United States Government. Neither the United States Government nor any agency thereof, nor any of their employees, makes any warranty, express or implied, or assumes any legal liability or responsibility for the accuracy, completeness, or usefulness of any information, apparatus, product, or process disclosed, or represents that its use would not infringe privately owned rights. Reference herein to any specific commercial product, process, or service by trade name, trademark, manufacturer, or otherwise does not necessarily constitute or imply its endorsement, recommendation, or favoring by the United States Government or any agency thereof. The views and opinions of authors expressed herein do not necessarily state or reflect those of the United States Government or any agency thereof. 


\section{DISCLAIMER}

Portions of this document may be illegible in electronic image products. Images are produced from the best available original document. 
Alternatively, the presence of two eigen polarizations for each spatial mode can be exploited for modulation between the orthogonal polarizations. The polarization modulation frequency ideally should not be limited by the carrier recombination lifetime and occur without chirp.

In this paper we review the gain-dependent polarization properties of VCSELs, and present techniques for the control and modulation of the VCSEL polarization. We first show that the power partitioning into the eigen polarizations is intrinsically related to the relative spectral overlap of the nondegenerate polarization cavity resonances with the laser gain spectrum. 12 We next describe techniques for controlling the VCSEL polarization of the fundamental and higher-order modes using laser gain offset, 12 anisotropic transverse cavity geometries, ${ }^{11}$ or the inherent material properties of the gain medium. ${ }^{13}$ Finally, we present two means for obtaining abrupt polarization switching transitions to enable polarization modulation. In particular using a cruciform VCSEL geometry, we demonstrate electrically driven polarization modulation, complementary digital polarized switching, and frequency doubling of the fundamental mode polarized output. 14

\section{VCSEL FABRICATION AND TESTING}

We have characterized VCSELs fabricated from many wafers at a number of emission wavelengths, including 980, 850, and $680 \mathrm{~nm}$. The infra-red VCSEL wafers are grown by molecular beam epitaxy (MBE) and/or metalorganic vapor phase epitaxy ${ }^{15}$ (MOVPE), while the visible VCSEL wafers are grown exclusively by MOVPE. 16 The VCSEL wafers are designed to emit from the top surface and consist of epitaxial distributed Bragg reflector (DBR) mirrors surrounding a 1- $\lambda$ thick active region containing InGaAs, GaAs, or GaInP quantum wells. The MBE wafers are grown on (100) substrates and are rotated during growth. The wafers grown by MOVPE are on misoriented (100) substrates tilted $6^{\circ}$ toward $<111>A$ or on $<311>$ oriented substrates. The MOVPE wafers are intentionally not rotated during growth to produce along the direction of gas flow a 5-7\% variation of layer thickness and thus cavity resonance wavelength, but only a $1 \%$ change in the peak gain wavelength. This wafer nonuniformity allows examination of VCSELs possessing a wide variation of spectral overlap between the peak laser gain and the cavity resonance wavelength as a function of the device position on the wafer.

The two VCSEL device structures considered in this paper are sketched in Fig. 1. Air-post VCSELs shown in Fig. 1(a) are fabricated by a self-aligned process using metal masks (of various shapes) with $\mathrm{SiO}_{2}$ windows protecting the laser aperture during reactive ion etching. The metal masks also serve as the top electrical contact. ${ }^{17}$ The air-post VCSELs are etched through the active region and partially into the lower DBR to provide electrical and index-guided optical confinement. Depicted in Fig. 1(b) are planar gain-guided VCSELs, which are fabricated using proton implantation to compensate the material around the laser cavity in order to funnel current into the active region. 18 The mask used during implantation thus defines the size and the transverse geometry of the laser cavity. All of the circular implanted VCSELs described herein have $15 \mu \mathrm{m}$ diameter cavities with $10 \mu \mathrm{m}$ diameter top contact apertures.

The VCSELs are tested on a probe station at heat sink temperatures varying from 100 to $350 \mathrm{~K}$. Polarization-resolved continuous wave light intensity versus injection current $(\mathrm{L}-\mathrm{I})$ measurements are made using a HP4156B semiconductor parametric analyzer and by positioning a linear polarizer in front of a calibrated Si photodetector. In the figures which follow, the directions labeled as $0^{\circ}$ and $90^{\circ}$ are parallel with the $<110>$ crystal axes of the VCSEL wafers; eigen polarizations labeled as $\perp$ and $\|$ are not aligned along the $<110>$ crystal directions. The spectral splittings between the eigen polarizations of the $850 \mathrm{~nm}$ VCSELs are measured using a scanning Fabry-Perot interferometer with a resolution of approximately 800 MHz. To test the polarization modulation characteristics, the lasers are operated with a sinusoidal AC voltage impressed on a DC bias level using a HP8116A function generator. 


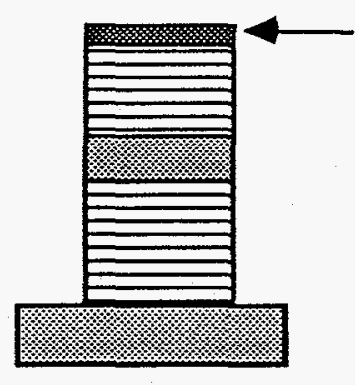

(a) Air-Post

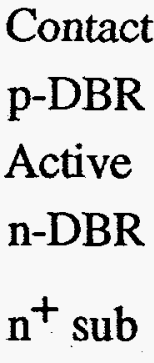

$\mathrm{n}-\mathrm{DBR}$
$\mathrm{n}^{+} \mathrm{sub}$

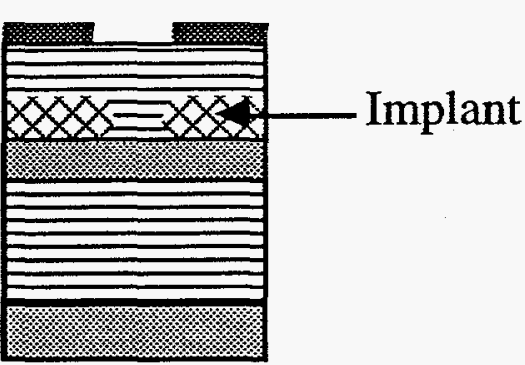

(b) Implanted

Figure 1. Sketches of (a) etched air-post and (b) implanted VCSELs.
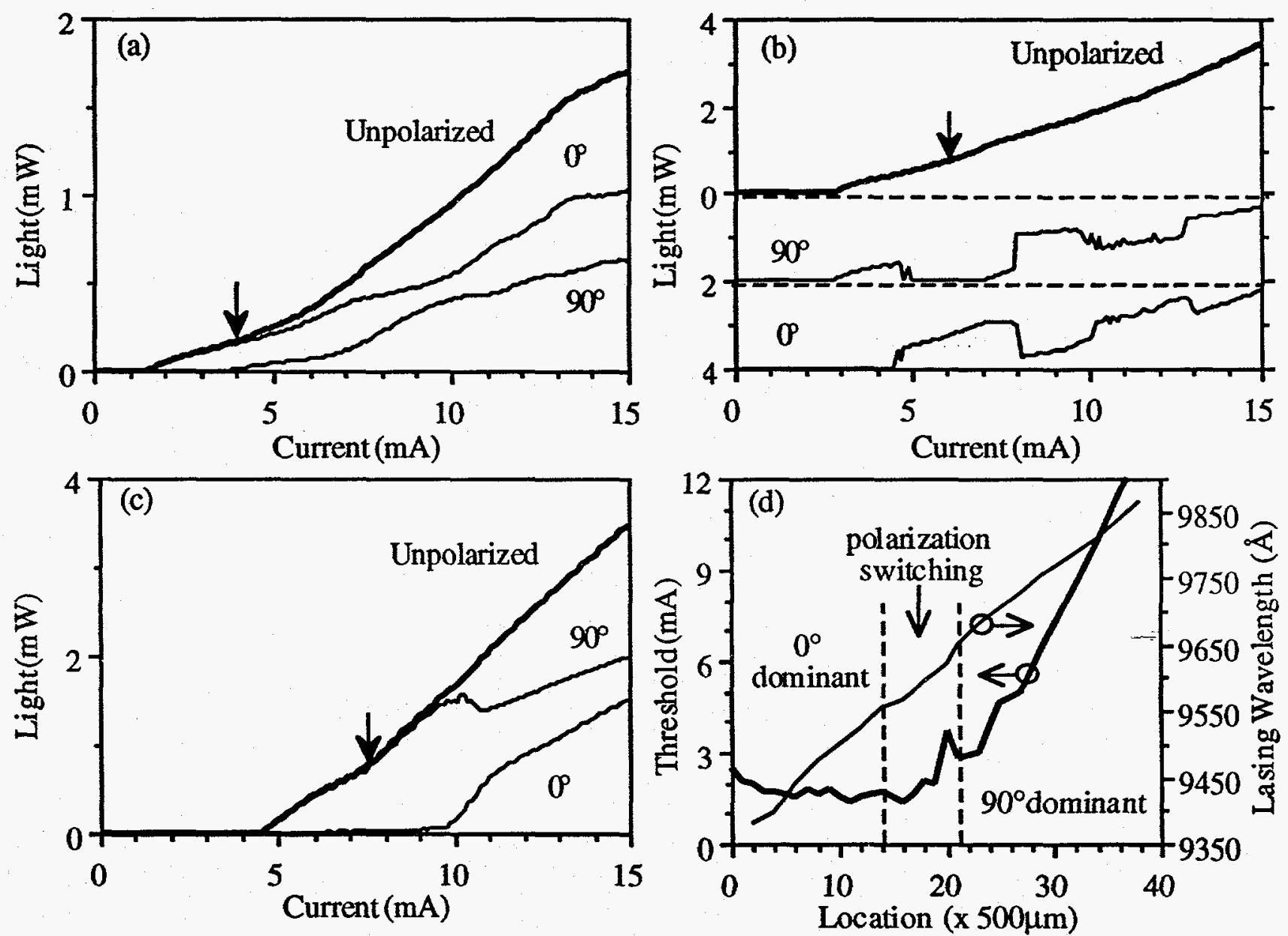

Figure 2. Polarization characteristics of $15 \mu \mathrm{m}$ diameter implanted InGaAs VCSELs. Unpolarized (thick curve) and polarized (thin curves) emission at different wafer locations: (a) $\lambda_{\text {th }}=943 \mathrm{~nm}$, (b) $\lambda_{\text {th }}=965 \mathrm{~nm}$ (curves offset for clarity), and (c) $\lambda_{\mathrm{th}}=970 \mathrm{~nm}$. The eigen polarizations are aligned along the $\langle 110\rangle$ crystal directions denoted as $0^{\circ}$ and $90^{\circ}$, and the arrows denote the onset of a higher-order transverse spatial mode. (d) $I_{\text {th }}$, wavelength, and dominant polarization at threshold versus location along the centerline of the wafer. 


\section{POLARIZATION GAIN-DEPENDENCE}

For VCSELs operating above lasing threshold, we typically observe the linearly polarized output to have an intensity maximum and minimum in orthogonal directions, corresponding to the TE eigen polarizations. The directions of the eigen polarizations are often aligned with the $\langle 110\rangle$ crystal axes, but can also be randomly oriented in the plane of the quantum wells. $1,2,4$ Fig. 2 shows polarized and unpolarized L-I curves of circular implanted InGaAs VCSELs from various locations on a wafer grown by MOVPE. 12 Notice in Fig. 2 both the laser peak gain/cavity resonance wavelength alignment and the dominant threshold polarization state vary with wafer location (the former due to the intentional wafer nonuniformity). We summarize in Fig. 2 (d) the threshold current, $\mathrm{I}_{\mathrm{th}}$, the threshold wavelength, and the dominant threshold eigen polarization versus location along the centerline of the wafer. In the region of the wafer with the shortest cavity resonance, the laser gain spectrum is red-shifted relative to the cavity resonances and the $0^{\circ}$ eigen polarization consistently dominates for all devices, as in Fig. 2(a). Conversely, at the opposite end of the wafer with blue-shifted gain, the $90^{\circ}$ eigen polarization consistently dominates as shown in Fig. 2(c). In between these regions, near the vicinity of the wafer with lasers exhibiting minimum $\mathbf{I}_{\text {th }}$, the $90^{\circ}$ state initially dominates until an abrupt switching transition where upon the $0^{\circ}$ eigen polarization prevails as displayed in Fig. 2(b).

Figure 3 depicts the temperature dependence of $\mathrm{I}_{\mathrm{th}}$ and the dominant threshold eigen polarization for a circular implanted GaAs VCSEL grown by MBE. 2 In Fig. 3 the spectral mismatch between the laser gain and the cavity resonance is varied by their noncommensurate thermal shifts ${ }^{19}$ rather than by layer thickness nonuniformity as in Fig. 2. We again observe that the eigen polarizations are each dominant at temperatures on opposite sides of the minimum $I_{t h}$, separated by a temperature region where the VCSEL exhibits polarization switching. The inset in Fig. 3 shows the spectral splitting at room temperature between the eigen polarizations of the fundamental mode, which are not parallel to the $\langle 110\rangle$ crystal axes. The two peaks, which correspond to the two eigen polarizations, are separated by $9.9 \mathrm{GHz}(0.23 \AA)$ which remains constant with current, voltage, or temperature changes, but can be varied by locally applied strain. 12

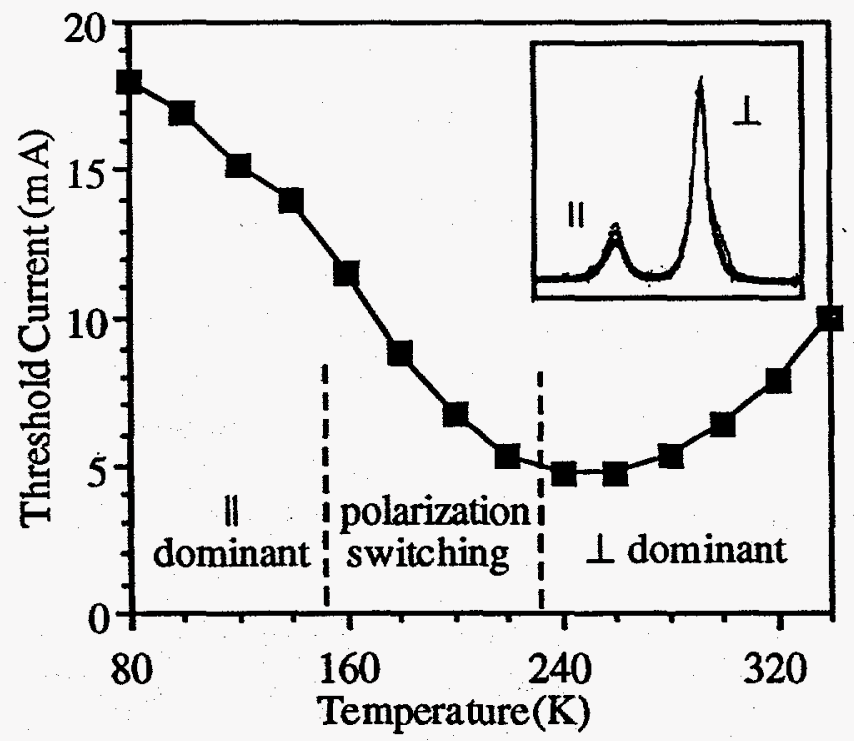

Figure 3. Ith and dominant threshold polarization at threshold for a $15 \mu \mathrm{m}$ diameter implanted $\mathrm{GaAs}$ VCSEL. The eigen polarizations, denoted as $\perp$ and $\|$, are not aligned along the $\langle 110\rangle$ crystal directions. The inset shows the room temperature spectral splitting $(9.9 \mathrm{GHz})$ between the eigen polarizations of the fundamental mode. 


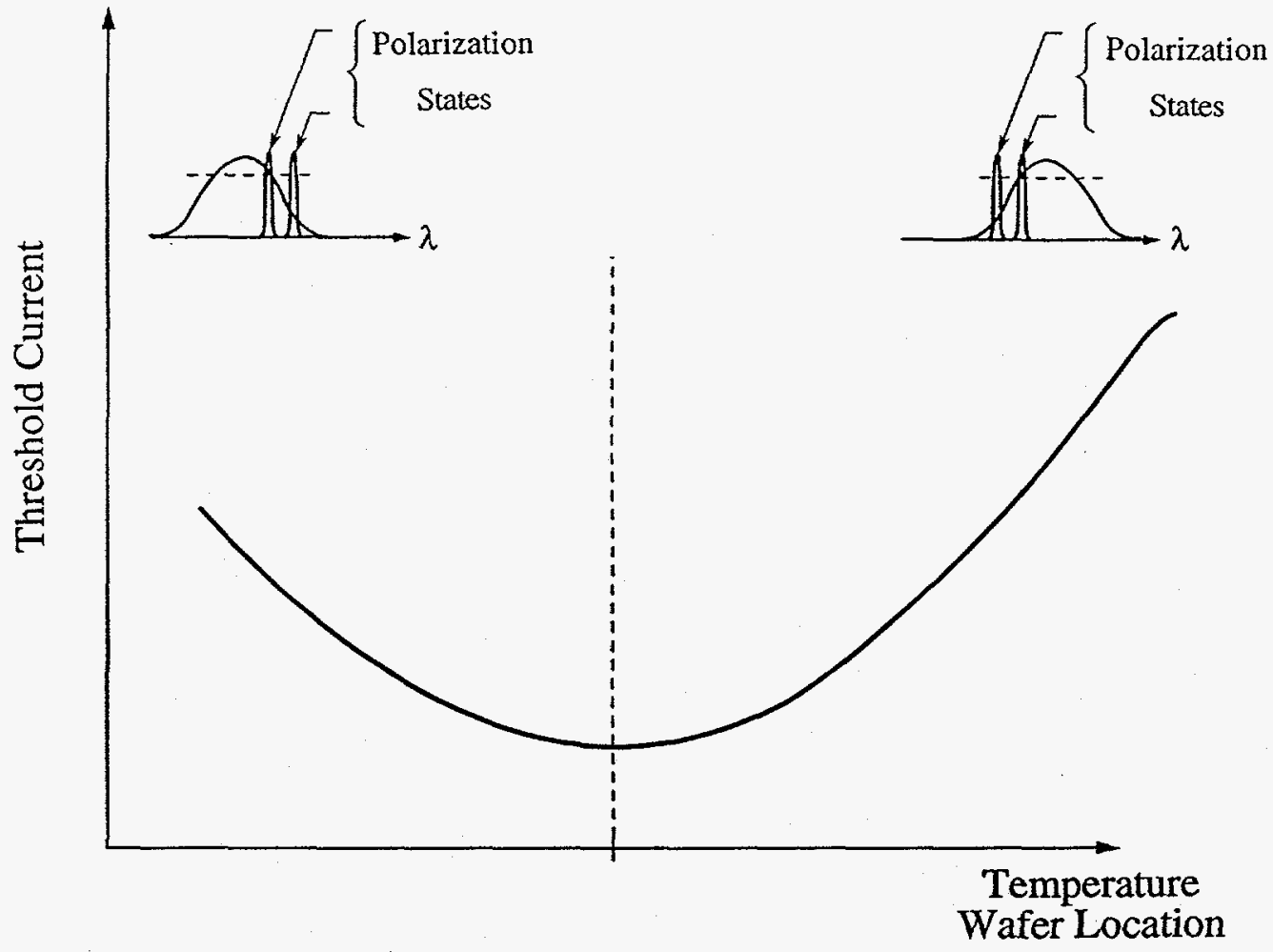

Figure 4. Schematic of $I_{t h}$ vs. substrate temperature or wafer location (not to scale). The insets depict the relative spectral alignment of the laser gain with the nondegenerate eigen polarization cavity resonances of the fundamental mode (splitting greatly exaggerated) which produces the polarization gain-dependence.

The polarization power partitioning and the $\mathrm{I}_{\text {th }}$ dependence on wafer location or substrate temperature are both related to the relative spectral alignment of the cavity resonances with the laser gain. In Figs. 2(d) and 3 the minimum $I_{\text {th }}$ occurs at the wafer location or substrate temperature where the cavity resonance and the peak laser gain are optimally aligned. When the gain is blue- or red-shifted relative to the cavity resonance, greater injection is required to achieve lasing. Due to the spectral splitting of the fundamental mode eigen polarizations, under the condition of blue-shifted gain, the shorter wavelength eigen polarization will experience enhanced gain overlap, while for red-shifted gain, the longer wavelength polarization state will dominate. This is schematically depicted in Fig. 4, where the insets show the differing gain alignment with the nondegenerate polarization cavity resonances. Moreover, near the condition of cavity resonance/peak gain alignment, the dominant polarization abruptly switches as the redshifting gain sweeps through the polarization resonances due to ohmic heating with increasing current injection. Such polarization switching is apparent for the fundamental mode and higher-order transverse modes in Fig. 2(b). Note that VCSEL designs which incorporate optimum gain/cavity resonance alignment to achieve high output power and greater temperature insensitivity ${ }^{20}$ also create the precise circumstance for polarization switching transitions.

This gain-dependence of the VCSEL polarization requires sufficient spectral splitting between the polarization cavity resonances to produce enough difference of gain to allow one or the other eigen polarization to dominate. For GaAs VCSELs we empirically observe the polarization gain-dependence when the eigen polarizations are separated by $>3 \mathrm{GHz}(>0.07 \AA) .12$ If the splitting is less than this, the polarization states often have equal or nearly equal intensity. We further find that the eigen polarization splitting varies only slightly between VCSELs from a given sample; however, the splitting can significantly differ between different samples even when from the same wafer. Since locally applied stress influences the spectral splitting, residual stress in the epilayers resulting from growth or device fabrication may produce the birefringence between the eigen polarizations. 


\section{POLARIZATION CONTROL}

The polarization gain-dependence and the random orientation of the eigen polarizations can conspire to produce polarization partitioning noise ${ }^{2}$ and/or an unspecified polarization state in the VCSEL output. Thus VCSEL polarization control requires maintaining a single polarization state in a specific direction. Note that neither misoriented substrates nor strained quantum wells can guarantee polarization alignment along the $\langle 110\rangle$ crystal axes, 21 although this is sometimes found. Strategies to produce a dominant eigen polarization usually use anisotropic gain 7,8 or loss 9,10 in the laser. With differing degrees of success, these techniques promote a single polarization for the fundamental mode, but at the expense of increased fabrication complexity, and higher-order modes still tend to remain orthogonally polarized. We describe in the following other methods of obtaining a single dominant polarization for the fundamental and higher-order modes for all VCSELs in a wafer.

In the preceding section we showed that one or the other eigen polarization will dominate near threshold when the peak laser gain is offset from the cavity resonances. Fig. 2 (c) illustrates that with a sufficient blue-shift of the gain, the first higherorder mode will also consistently arise (denoted at the arrows in Fig. 2) with the same polarization as the fundamental. We find the number of transverse modes possessing the same polarization as the fundamental increases as the blue-shifted gain offset is increased above $15-20 \mathrm{~nm}$ in our InGaAs VCSELs. 12 This indicates that spatial/spectral hole burning is of less consequence with blue-shifted gain, and/or the asymmetry of the gain spectrum affects the polarization partitioning of higher-order modes. Therefore, gain-guided VCSEL arrays with each element having its peak gain blue-shifted $\geq 20$ $\mathrm{nm}$ from the cavity resonance will provide a single dominant polarization through the onset of multimode operation. As an aside, note that for VCSEL array polarization uniformity, it is crucial that the polarization cavity resonances do not span the gain peak maximum in order to avoid polarization switching evident in Fig. 2(b) and the concomitant polarization noise.

Another means to influence the VCSEL polarization is through the geometry of the laser cavity. 11 Analogous with the development of
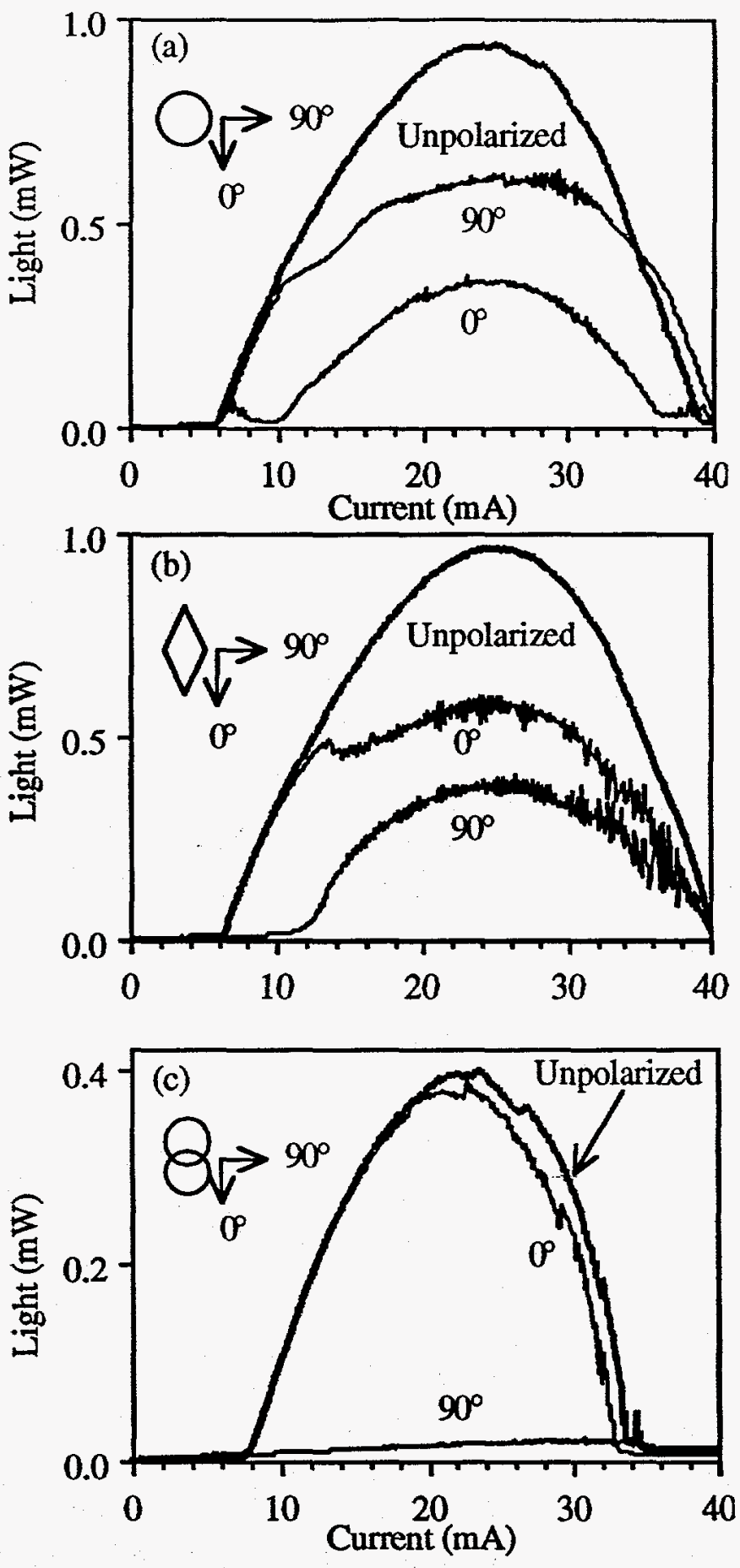

Figure 5. Unpolarized (thick curves) and polarized (thin curves) emission from $\mathrm{GaAs}$ air-post VCSELs with various geometries: (a) $20 \mu \mathrm{m}$ diameter circular laser; (b) rhombus-shaped laser; and (c) dumbbell-shaped laser. 

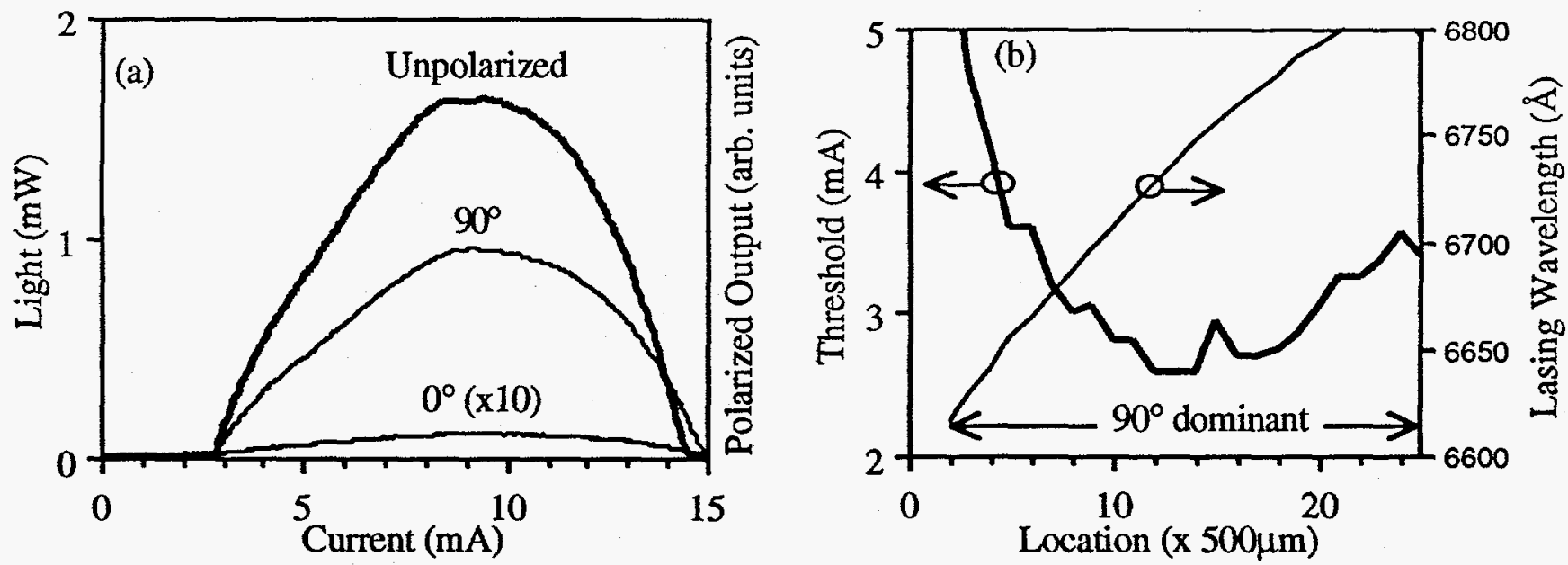

Figure 6. (a) Unpolarized (thick curve) and polarized (thin curves) emission from a $15 \mu \mathrm{m}$ diameter implanted GaInP visible VCSEL; (b) $\mathrm{I}_{\mathrm{th}}$, wavelength, and dominant polarization at threshold versus wafer location.

single-polarization optical fiber, 22 it is expected that noncircular VCSEL cavities will influence the polarization properties. Fig. 5 shows the unpolarized and polarized L-I curves for GaAs air-post VCSELs with circular, rhombus-shaped, and dumbbell-shaped cross sections with equal cross section areas. Notice for the circular VCSEL in Fig. 5(a), above $8 \mathrm{~mA}$ the $90^{\circ}$ eigen polarization is dominant for the fundamental mode, which is also found for the other circular VCSELs in this sample. Above 10mA a higher order transverse mode begins to lase with an orthogonal polarization. For the rhombus VCSEL shown in Fig. 5(b), the $0^{\circ}$ eigen polarization, parallel to the major axis of the anisotropic cavity, dominates the lasing emission over the entire operating range. The dominant eigen polarization of air-post rhombus VCSELs is always found to be oriented along the major axis of the cavity when parallel to a $<110>$ crystal axis. The dominant $0^{\circ}$ eigen polarization of the dumbbell VCSEL depicted in Fig. 5(c) is also found to be parallel with the major axis of the laser cavity, again along a $<110>$ crystal axis. In addition, the lasing emission polarized orthogonally is reduced; at maximum output power in Fig. 5(c), power in the $90^{\circ}$ polarization is suppressed by $14 \mathrm{~dB}$ relative to the $0^{\circ}$ eigen polarization. It is likely that higher loss for the $90^{\circ}$ polarization is introduced by the constricted region at the center of the dumbbell-shaped cavity providing the polarization discrimination. Comparing Figs. 5(a) with 5(b) and 5(c) we see the dominant eigen polarization of the isotropic circular VCSEL can be rotated by $90^{\circ}$ using the rhombusor dumbbell VCSEL designs. This demonstrates that a single eigen polarization in a specified crystal direction can be selected and maintained using anisotropic VCSEL geometries.

Finally, we conclude by reviewing the polarization characteristics of GaInP visible VCSELs. ${ }^{13}$ In Fig. 6(a) we show typical unpolarized and polarized L-I curves for a 1- $\lambda$ cavity circular implanted VCSEL ${ }^{23}$ which is $15 \mu \mathrm{m}$ in diameter and emits at $680 \mathrm{~nm}$. In Fig. 6(b) (which is analogous to Fig. 2(d)) we plot $I_{t h}$, threshold wavelength, and the dominant eigen polarization observed corresponding to differing peak gain/cavity resonance alignment across the wafer. For all GaInP VCSELs examined from this wafer and others, the dominant eigen polarization is always aligned along one particular $<110\rangle$ crystal axis and is consistently dominant for all transverse lasing modes as shown in Fig. 6(a). Furthermore this eigen polarization dominates independent of the laser gain alignment (see Fig. 6(b)) and independent of operating temperature. 13 By comparison to infra-red VCSELs, we can discount the effects of substrate misorientation, thickness nonuniformity, or the quantum well strain to account for the polarization control. It is likely that the inherent material properties of the InGaP active region (i.e. sublattice ordering) contributes to the profound polarization control and is currently under investigation. A predetermined and fixed polarization for all visible VCSELs on a wafer over a wide temperature range and for all lasing modes may prove valuable for polarization-sensitive applications. 


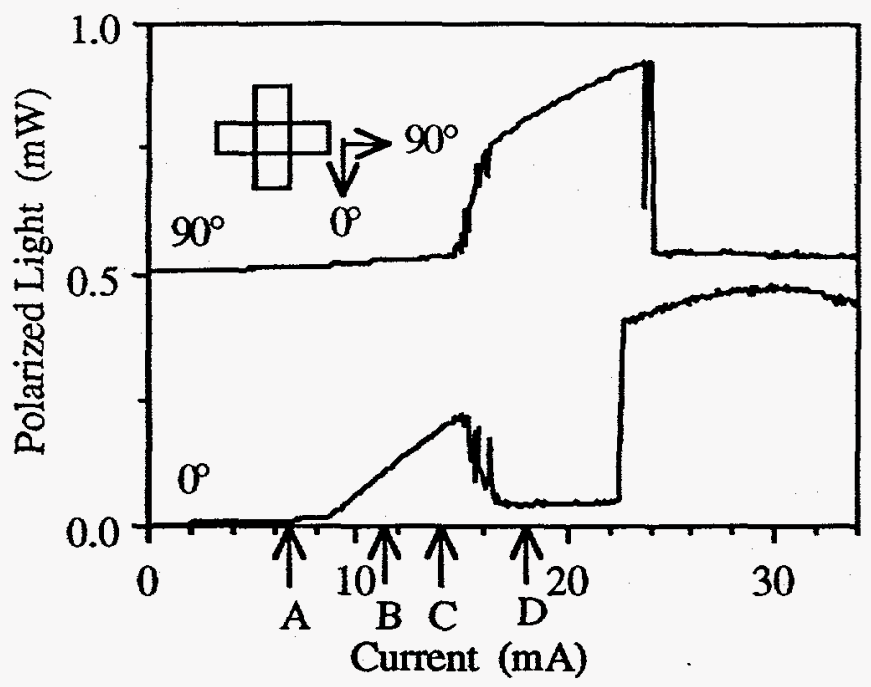

Figure 7. Polarized emission (curves offset for Fig. 8. Small signal modulation for an air-post

clarity) from a cruciform air-post VCSEL. The cruciform VCSEL at $50 \mathrm{MHz}$ : (a) input waveform; points denoted along the abscissa correspond to current modulation limits described in Figs. 8 and 9.

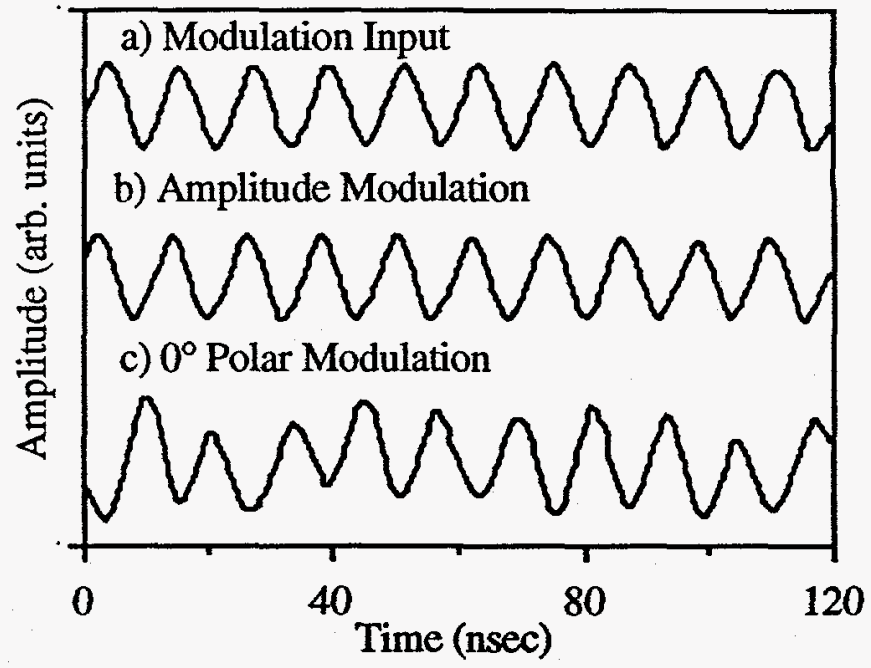
(b) $0^{\circ}$ output where the current varies between points $\mathrm{B}$ and $\mathrm{C}$ in Fig. 7; and (c) $0^{\circ}$ output where the current varies about a polarization switching transition, i.e. points $\mathrm{C}$ and $\mathrm{D}$ in Fig. 7.

\section{POLARIZATION MODULATION}

Instead of controlling the VCSEL polarization, in this section we seek to exploit the interaction between the eigen polarizations. Polarization modulation is demonstrated where a specific spatial transverse mode is maintained, but its polarization state is varied with a small modulation signal. ${ }^{14}$ Hence, the switching of the polarized output may not be limited by the carrier recombination lifetime, since the lasing inversion is constantly maintained, and the modulation should occur with little or no chirp in the output light. Inducing polarization switching in VCSELs at GHz frequencies has been accomplished through polarized optical feedback. 24,25 In the following we describe the first electrically driven polarization modulation ${ }^{14}$ using polarization switching transitions induced by two different techniques.

In Section 3, we showed that near the condition of laser gain/cavity resonance alignment, the dominant polarization switches as the gain sweeps through the polarization cavity resonances. Thus by biasing a suitable VCSEL into such a polarization switching transition, such as found in Fig. 2(b), a small variation of the injection current will switch the polarized output between the two eigen polarizations. In this case the polarization switching is produced by the thermal shift of the laser gain (i.e. band gap) and thus is a relatively slow process. Experimentally we find the maximum polarization modulation of suitable circular gain-guided InGaAs VCSELs to be limited to $\leq 80 \mathrm{kHz} .{ }^{14}$

A second means of achieving a polarization switching transition is through transverse mode engineering. In Fig. 7 we show the polarized L-I curves for an air-post GaAs VCSEL with a cruciform cross section geometry. ${ }^{14}$ A single lasing spectral peak is observed over the entire operating range of this laser, indicative of lasing only in the lowest-order spatial mode. However, two polarization switching transitions between the eigen polarizations are evident in Fig. 7. In Fig. 8 we present the small signal polarization modulation characteristics of an air-post cruciform VCSEL. Fig. 8(a) depicts the input AC voltage waveform at $50 \mathrm{MHz}$; the resultant $0^{\circ}$ polarized output is shown in Fig. 8(b) which corresponds to amplitude modulation between points B and C in Fig. 7. Fig. 8(c) shows the $0^{\circ}$ polarized output where the input current is modulated through the first polarization transition, corresponding to points $C$ and $D$ in 

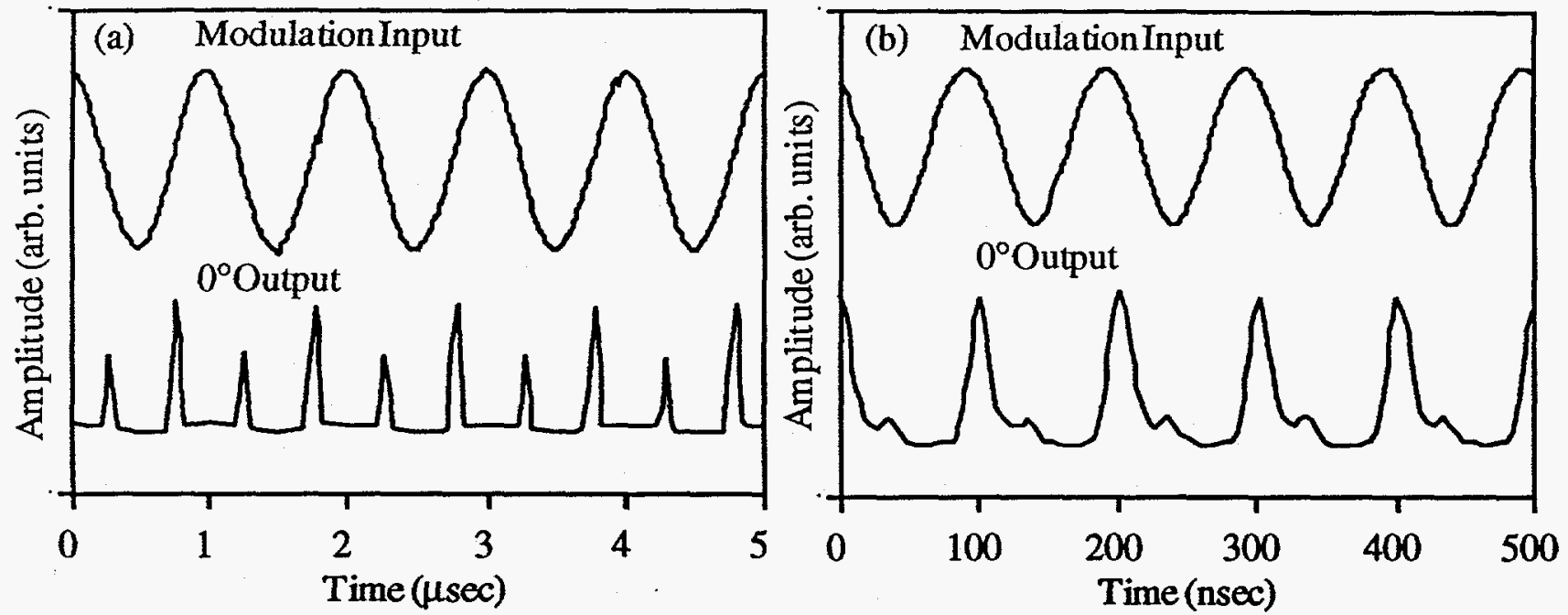

Figure 9. Large signal modulation characteristics for an air-post cruciform VCSEL: (a) input voltage waveform at $1 \mathrm{MHz}$ and resultant frequency doubled $0^{\circ}$ output where the injection current varies from below threshold to above the polarization switching transition, such as points A and D in Fig. 7; (b) input voltage waveform at $10 \mathrm{MHz}$ and resultant frequency doubled $0^{\circ}$ output.

Fig. 7. In Fig. 8(c) the polarized output decreases as the input injection current increases, and thus shifts in phase by $180^{\circ}$ with respect to the amplitude modulation in Fig. 8(b), confirming polarization modulation.

Figure 9 depicts the polarized output behavior of the cruciform air-post VCSEL under large signal modulation. ${ }^{14}$ The top curve in Fig. 9(a) shows the input AC voltage waveform at $1 \mathrm{MHz}$, corresponding to modulation from below threshold to above the polarization transition, points $A$ and $D$, respectively, in Fig. 7. In this manner a $0^{\circ}$ polarized output signal at twice the input frequency can be generated as shown in the lower curve in Fig. 9(a). The two output peaks per input cycle arise from the cessation of lasing as the current goes below threshold and from switching to the $90^{\circ}$ polarization as the current goes above the polarization switching transition. As the input frequency increases, the intensity of one of the polarized output peaks decreases, and, as evident in the lower curve in Fig. 9(b), ultimately dišappears for modulation frequencies $\geq 10 \mathrm{MHz}$. Multiple polarization switching transitions have also been observed from implanted gain-guided cruciform VCSELs as shown in Fig. 10. By adjusting the amplitude of the AC drive voltage, complementary polarized digital switching shown in Fig. 11(a), or frequency doubling of both eigen polarizations shown in Fig. 11(b), is obtained.

The polarization switching transitions of the cruciform VCSELs are thought to arise from interactions between the eigen polarizations rather than the laser gain/cavity resonance overlap. For the lasers shown in Figs. 7 and 10, the gain is always red-shifted relative to the cavity resonance so spectral alignment does not occur during operation of these VCSELs. It appears that the cruciform geometry equalizes the gain of the two orthogonal eigen polarizations or at least modifies the gain competition between them. Because the cross-shaped gain region is uniformly pumped, the polarization switching may arise from spatial and/or spectral hole burning between the eigen polarizations. As gain is clamped for one polarization, carrier inversion increases for the orthogonal polarization leading to its eventual lasing. In any event, the maximum polarization modulation frequency of cruciform VCSELs, although faster than the thermallylimited response of the circular VCSELs, is found to be limited to the few 10 's of MHz regime. 


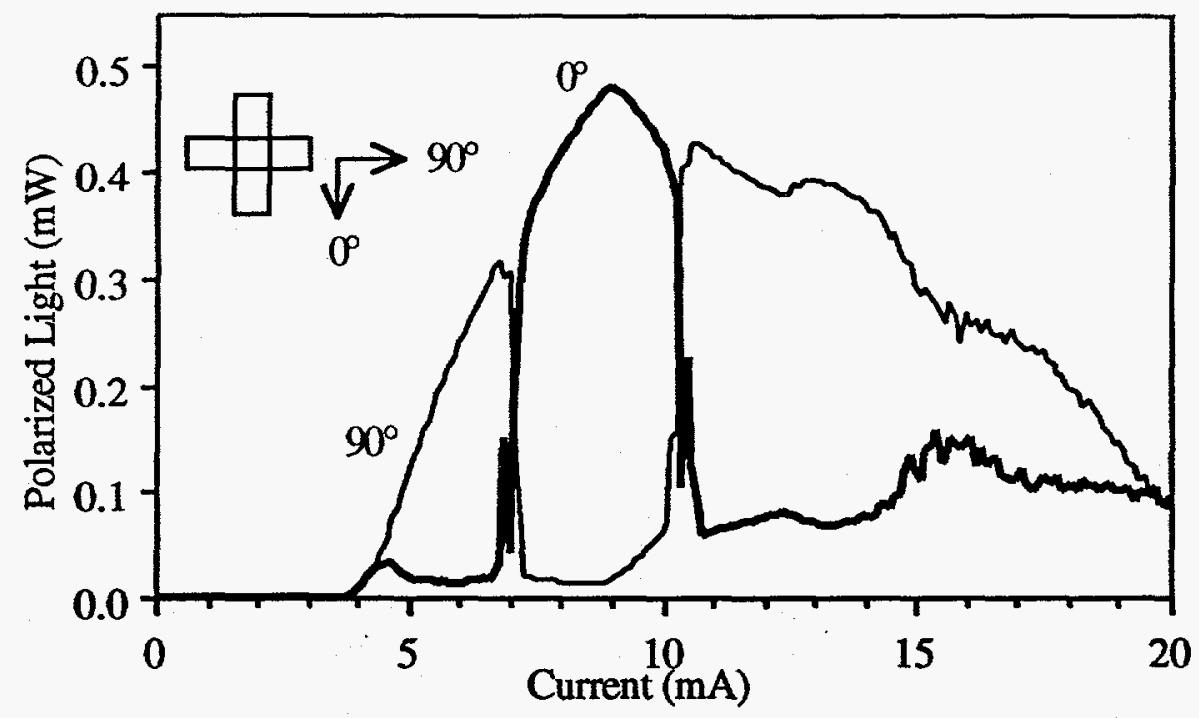

Fig. 10. Polarized emission from an implant cruciform VCSEL; notice the multiple polarization transitions between the $0^{\circ}$ (thick curve) and $90^{\circ}$ (thin curve) eigen polarizations.
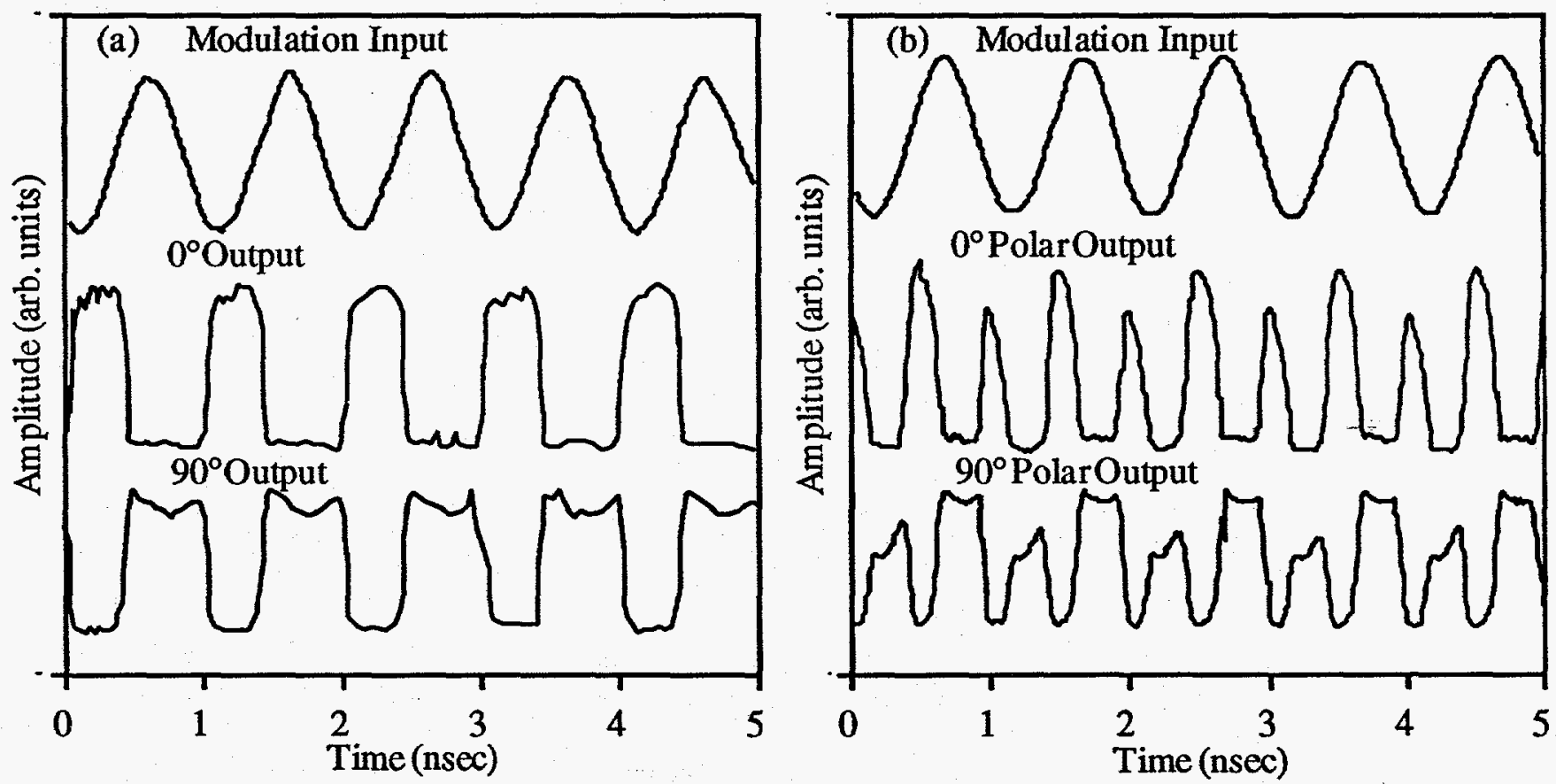

Fig. 11. Polarization modulation characteristics for an implanted cruciform VCSEL at $1 \mathrm{MHz}$ : (a) complementary binary polarized output obtained by small signal modulation of the input about the first polarization switching transition in Fig. 10; and (b) frequency doubling of the output for both eigen polarizations by large signal modulation of the input about the two polarization switching transitions in Fig. 10. 


\section{CONCLUSIONS}

The polarization gain-dependence, polarization control, and polarization modulation of VCSELs have been reviewed. The power partitioning between the eigen polarizations is correlated with the spectral alignment of the nondegenerate polarization cavity resonances with the laser gain spectrum. Since this gain-dependence requires sufficient spectral splitting between the eigen polarizations, some means to engineer this splitting to influence the gain-dependence are under investigation. At the condition where the peak gain and the cavity resonance are aligned, abrupt polarization switching and polarization noise can occur. The polarization gain-dependence does allow polarization control through sufficient blue-shifting of the gain to select the dominant eigen polarization. Therefore, careful control of the VCSEL cavity resonance/gain alignment will be necessary to achieve both high-performance and polarization control.

The VCSEL polarization can also be controlled by the transverse geometry of the laser cavity. Whereas circular VCSELs can have competing orthogonal polarizations, anisotropic transverse cavity designs, such as rhombus or dumbbell shapes, can select a dominant eigen polarization. The most stringent polarization control has been observed in visible GaInP VCSELs, where a fixed orientation and a single dominant polarization state may be intrinsic to the active medium. Finally, exploiting the presence of the two VCSEL eigen polarizations enables polarization modulation. Electrically controlled polarization modulation of cruciform VCSELs to frequencies as high as $50 \mathrm{MHz}$ is demonstrated, along with other novel manipulations of the polarized output. Polarization modulation at higher frequencies is desirable, but will likely require an alternative switching mechanism. Nevertheless, the present polarization modulation could find application as logic states in optical computing applications. Therefore, understanding, controlling, and exploiting the unique polarization attributes of VCSELs will be advantageous for their deployment in present and future system applications.

\section{ACKNOWLEDGMENTS}

The authors thank S. Samora, R. C. Wetzel, ${ }^{*}$ R. J. Shul, J. Escobedo, and K. M. Geib for technical assistance during the course of this research. The work performed at Sandia National Laboratories is supported by the U. S. Department of Energy under contract No. DE-AC04-94AL85000.

\section{REFERENCES}

${ }^{1}$ C. J. Chang-Hasnain, J. P. Harbison, L. T. Florez, and N. G. Stoffel, "Polarization characteristics of quantum well vertical cavity surface emitting lasers," Electron. Lett. 27, pp. 163-165 (1991).

${ }^{2}$ K. D. Choquette, D. A. Richie, and R. Leibenguth, "Temperature dependence of gain-guided verticalcavity surface emitting laser polarization," Appl. Phys. Lett. 64, pp. 2062-2064 (1994).

${ }^{3}$ F. S. Choa, Y. H. Lee, T. L. Koch, C. A. Burrus, B. Tell, J. L. Jewell, and R. E. Leibenguth, "High speed modulation of vertical-cavity surface emitting lasers," IEEE Photon. Technol. Lett. 3, pp. 697699 (1991).

4J. L. Jewell, S. L. McCall, Y. H. Lee, A. Scherer, A. C. Gossard, and J. H. English, "Lasing characteristics of GaAs microresonators," Appl. Phys. Lett. 54, pp. 1400-1402 (1989).

${ }^{5}$ C. J. Chang-Hasnain, J. P. Harbison, G. Hasnain, A. C. Von Lehmen, L. T. Florez, and N. G. Stoffel, "Dynamic, polarization, and transverse mode characteristics of vertical cavity surface emitting lasers," IEEE J. Quantum Electron. 27, pp. 1402-1408 (1991).

6D. Vakhshoori, "Symmetry considerations in vertical-cavity surface emitting lasers: prediction of removal of polarization isotropicity on (001) substrates," Appl. Phys. Lett. 65, pp. 259-261 (1994).

${ }^{7}$ T. Mukaihara, F. Koyama, and K. Iga, "Engineered polarization control of GaAs/AlGaAs surfaceemitting lasers by anisotropic stress from elliptical etched substrate hole," IEEE Photon. Technol. Lett. 5, pp. 133-135 (1993). 
${ }^{8}$ A. Chavez-Pirson, H. Ando, H. Saito, and H. Kanbe, "Polarization properties of a vertical cavity surface emitting laser using a fractional layer superlattice gain medium," Appl. Phys. Lett. 62, pp. 3082-3084 (1993).

${ }^{9}$ M. Shimuzi, T. Mukaihara, F. Koyama, and K. Iga, "Polarization control for surface emitting lasers," Electron. Lett. 27, pp. 1067-1069 (1991).

${ }^{10} \mathrm{~T}$. Mukaihara, N. Ohnoki, T. Baba, F. Koyama, and K. Iga, "A novel birefringent distributed Bragg reflector using a metal/dielectric polarizer for polarization control of surface emitting lasers," $J p n . J$ Appl. Phys. 33, pp. L227-L229 (1994)

${ }^{11} \mathrm{~K}$. D. Choquette and R. E. Leibenguth, "Control of vertical-cavity laser polarization with anisotropic transverse cavity geometries," IEEE Photon. Technol. Lett. 6, pp. 40-42 (1994).

12K. D. Choquette, R. P. Schneider, Jr., K. L. Lear, and R. E. Leibenguth, "Gain-dependent polarization properties of vertical-cavity lasers," IEEE Selected Topics of Quantum Electron. 1 (1995).

${ }^{13}$ K. D. Choquette, R. P. Schneider, Jr., and J. A. Lott, "Lasing characteristics of visible AlGaInP/AlGaAs vertical-cavity lasers," Opt. Lett. 19, pp. 969-971 (1994).

${ }^{14}$ K. D. Choquette, K. L. Lear, R. E. Leibenguth, and M. T. Asom, "Polarization modulation of cruciform vertical-cavity laser diodes," Appl. Phys. Lett. 64, pp. 2767-2769 (1994).

15K. L. Lear, R. P. Schneider, Jr., K. D. Choquette, S. P. Kilcoyne, J. J. Figiel, and J. Zolper, "Vertical-cavity surface emitting lasers with $21 \%$ efficiency by metalorganic vapor phase epitaxy," IEEE Photon. Technol. Lett. 6, pp. 1053-1055 (1994).

16R. P. Schneider, Jr., R. P.-Bryan, J. A. Lott, E. D. Jones, and G. R. Olbright, "MOVPE growth of InAlGaP-based visible vertical-cavity surface emitting lasers," J. Crystal. Growth 124, pp. 763-771 (1992).

${ }^{17}$ K. D. Choquette, G. Hasnain, Y. H. Wang, J. D. Wynn, R. S. Freund, A.Y. Cho, and R. E. Leibenguth, "GaAs surface emitting lasers fabricated by reactive ion etching," IEEE Photon. Technol. Lett. 3, pp. 859-862 (1991).

18Y. H. Lee, B. Tell, K. Brown-Goebeler, J. L. Jewel, and J. V. Hove, "Top surface emitting GaAs four quantum well lasers emitting at $0.85 \mu \mathrm{m}$," Electron. Lett. 26, pp. 710-711 (1990).

${ }^{19}$ B. Tell, K. F. Brown-Goebler, R. E. Leibenguth, F. M. Baez, and Y. H. Lee, "Temperature dependence of GaAs-AlGaAs vertical-cavity surface emitting lasers," Appl. Phys. Lett. 60, pp. 683-685 (1992).

20D. B. Young, J. W. Scott, F. H. Peters, M. G. Peters, M. L. Majewski, B. J. Thibeault, S. W. Corzine, and L. A. Coldren, "Enhanced performance of offset-gain high-barrier vertical-cavity surface emitting lasers," IEEE J. Quantum. Electron. 29, pp. 2013-2021 (1993).

${ }^{21}$ K. D. Choquette, R. P. Schneider, Jr., and R. Leibenguth, unpublished.

22V. Ramaswamy, W. G. French, and R. D. Standley, "Polarization characteristics of noncircular core single-mode fibers," Appl. Opt. 17, pp. 3014-3017 (1978).

${ }^{23}$ R. P. Schneider, Jr., M. Hagerott-Crawford, K. D. Choquette, K. L. Lear, and J. J. Figiel, "Improved AlGaInP/AlGaAs red $(680 \mathrm{~nm})$ VCSELs employing C as the sole p-type dopant," to appear in Appl. Phys. Lett. (1995).

${ }^{24}$ S. Jiang, Z. Pan, M. Dagenais, R. A. Morgan, and K. Kojima, "High-frequency polarization selfmodulation in vertical-cavity surface emitting lasers," Appl. Phys. Lett. 63, pp. 3545-3547 (1993).

${ }^{25}$ N. Badr, I. H. White, M. R. T. Tan, Y. M. Houng, and S. Y. Wang, "Enhanced polarization selfswitching in a vertical-cavity surface emitting laser by gain saturation of transverse modes," Electron. Lett. 30, pp. 1227-1229 (1994). 